\title{
Co-culture of defined bacteria to degrade seven sulfonated aromatic compounds: efficiency, rates and phenotypic variations
}

\author{
Thomas Thurnheer, Alasdair M. Cook, and Thomas Leisinger \\ Department of Microbiology, Swiss Federal Institute of Technology, ETH-Zentrum, CH-8092 Zürich, Switzerland
}

Summary A co-culture, consisting of five defined bacteria [e.g., T. Thurnheer, T. Köhler, A. M. Cook, and T. Leisinger: J Gen Microbiol 132:1215-1220], was able to degrade at least seven substituted benzenesulfonic acids in continuous culture. HPLC, total organic carbon analyses and colourimetric analyses showed that the sulfonated compounds could be completely degraded to biomass, $\mathrm{SO}_{4}^{2-}, \mathrm{NH}_{4}^{+}$and $\mathrm{CO}_{2}$. The maximum observed degradation rate was $138 \mathrm{mg}$ of $\mathrm{C} / \mathrm{h} \cdot 1$. The five organisms were Alcaligenes sp. strain 0-1 (substrates benzenesulfonic acid, 4-methylbenzenesulfonic acid and 2-aminobenzenesulfonic acid), two Pseudomonas spp., strains T-2 (substrates 4-methylbenzenesulfonic acid and 4sulfobenzoic acid) and PSB-4 (substrate 4-sulfobenzoic acid) and two unidentified rods, strains M-1 (substrates benzenesulfonic acid, 4-methylbenzenesulfonic acid and 3-aminobenzenesulfonic acid) and S-1 (substrates 4-aminobenzenesulfonic acid and 4-hydroxybenzenesulfonic acid). The system was operated for over 18 months with five sulfonates, and no competition was detected amongst the four organisms present, because all organisms were still present $(100 \%$ of the population after 7 months, 55\% after 18 months). Many bacteria isolated from the continuous culture after 18 months showed substrate ranges different from those of the original strains. The most common occurrence (33\% of the population) was the appearance of organisms which could degrade 2-aminobenzenesulfonic acid and 4-sulfobenzoic acid. Several cases of the loss of a character were seen but only rarely $(1 \%)$ was a net gain of characters observed. After 30 months, only two (of five) parents were present (35\% of the

Offprint requests to: A. M. Cook population) and some isolates could utilize all seven substrates on solid media.

\section{Introduction}

Substituted benzenesulfonates can be released to the environment as waste-products from the manufacture of dyestuffs, alkylbenzenesulfonates and wood pulp. Sulfonated aromatic compounds are seldom synthesized in Nature (Herbert and Holliman 1964), and have been described as nonbiodegradable (Bretscher 1981). The presence of a sulfonate group on the aromatic ring makes biodegradation difficult, and analogues with a carboxy group in place of the sulfonate group can be degraded readily (Leidner et al. 1980).

Benzenesulfonate and toluenesulfonate, considered non-biodegradable in 1948 (Czekalowski and Skarzynsky 1948), are now known to be readily degradable by microorganisms (Ghisalba 1983; Swisher 1987). Malaney (1960) and Symons and Del Valle-Rivera (1961) observed oxygen uptake by resting cells of bacteria in the presence of 3- and 4-aminobenzenesulfonates but no data on mineralization of aminobenzenesulfonates was available till we demonstrated the complete degradation of substituted benezenesulfonates by pure cultures (Thurnheer et al. 1986).

The biodegradation of mixtures of substrates is important because industrial wastes are frequently mixtures of compounds. One approach to the problem is the genetic construction of strains with broad substrate ranges in vitro (Ramos and Timmis 1987). The behaviour of such strains with mixed substrates and their stability is uncertain, and the regulation of their use is still strict (Liberman et al. 1986). We have used another approach, 
co-cultures of organisms isolated from the environment, to explore the interactions amongst organisms and substrates (e.g., Bull and Slater 1982). A further problem with the biodegradation of industrial wastes is their fluctuation and their poor characterization. We have avoided this by using a defined mixture of determinable compounds.

We now demonstrate the complete mineralization of seven substituted benzenesulfonates by a defined co-culture in a chemostat and the appearance of new strains with wider substrate ranges than those of the original organisms.

\section{Materials and methods}

Chemicals. Most benzenesulfonates were purchased from Fluka (Buchs, Switzerland). 3-Aminobenzenesulfonate, 4-sulfobenzoate and 4-chlorobenzenesulfonate were obtained from Eastman (Rochester, NY), Ciba-Geigy AG. (Basel, Switzerland) and TCI (Tokyo, Japan), respectively. The sulfonates were chromatographically pure and their identity was confirmed as previously described (Thurnheer et al. 1986). All other chemicals were of reagent grade or better.

Analytical methods. The substituted benzenesulfonates were determined by high-pressure liquid chromatography (HPLC) after separation on a $\mathrm{C}_{18}$ reverse-phase column (Grossenbacher et al. 1986). Dissolved organic carbon was determined by standard methods (Greenberg et al. 1981) after removal of particulates by filtration. Protein in whole cells was measured as described by Cook and Hütter (1981).

Microorganisms, growth medium and culture conditions. The organisms used and their substrate ranges are shown in Table 1.

Table 1. Range of sulfonated compounds mineralized by the pure cultures

\begin{tabular}{lllllll}
\hline Carbon source & \multicolumn{5}{c}{ Organism $^{\mathrm{a}}$} \\
& M-1 & O-1 & PSB-4 & S-1 & T-2 \\
\cline { 2 - 6 } & & \multicolumn{3}{c}{ Substrate utilization } \\
\hline Benzenesulfonate (BS) & + & + & - & - & - \\
2-Aminobenzenesulfonate (OS) & - & + & - & - & - \\
3-Aminobenzenesulfonate (MS) & + & - & - & - & - \\
4-Aminobenzenesulfonate (SS) & - & - & - & & + & - \\
4-Carboxybenzenesulfonate (SB) & - & - & + & - & + \\
4-Hydroxybenzenesulfonate (PS) & - & - & - & & + & - \\
4-Methylbenzenesulfonate (TS) & + & + & - & - & +
\end{tabular}

a The bacteria were isolated by Thurnheer et al. (1986) and H. Locher, T. Thurnheer, T. Leisinger and A. M. Cook (in preparation). Three organisms were identified: Alcaligenes denitrificans $\mathrm{O}-1$ and Pseudomonas testosteroni $\mathrm{T}-2$ and PSB-4 (G. Auling, personal communication). Strains M-1 and $S-1$ were not identified

b (+) growth and complete disappearance of substrate within 10 days; (-) no growth and no degradation in 10 days
The mineral medium was described by Thurnheer et al. (1986) and contained 1 to 8 sulfonated compound(s) as carbon source(s) in concentrations of 1 to $9 \mathrm{mM}$ : when dilution rates were above $0.020 \mathrm{~h}^{-1}$, the concentrations of $\mathrm{MgSO}_{4}, \mathrm{NH}_{4} \mathrm{Cl}$ and trace elements were doubled. Solid media were prepared by addition of $1.5 \%$ agar to growth medium containing the appropriate carbon source.

The co-culture was grown in a chemostat with a working volume of 21 (MBR, Wetzikon, Switzerland); the temperature was set at $30^{\circ} \mathrm{C}, \mathrm{pH}$ at 7.3 , the stirrer at 500 to $1500 \mathrm{rpm}$ and the system was aerated with compressed air ( 1 to $21 / \mathrm{min}$ ). The chemostat was operated under aseptic conditions; the introduction of contaminants while cleaning electrodes was possible, but all isolates from the chemostat could degrade a sulfonated aromatic compound. The chemostat was first inoculated with $50-\mathrm{ml}$ batch cultures of strains $0-1$ and PSB-4 in medium containing 2-aminobenzenesulfonate, 4-sulfobenzoate and 4aminobenzenesulfonate. As soon as the sulfonated compounds in the chemostat had been degraded, the medium was fed continuously at dilution rates of 0.001 to $0.072 \mathrm{~h}^{-1}$. Samples $(20 \mathrm{ml})$ were taken at intervals, and substrate disappearance and the concentrations of dissolved organic carbon and of protein were measured.

Recovery of the single strains in the defined mixed culture. At 220,440 and 810 days after inoculation, a sample was taken, diluted with sterile, $0.9 \%(\mathrm{w} / \mathrm{v}) \mathrm{NaCl}$ solution and streaked on mineral-salts agar containing $5 \mathrm{mM}$ succinate. After 3-4 days of incubation at $30^{\circ} \mathrm{C}, 200$ single colonies were transferred to a set of mineral-salts agar plates each containing an aromatic sulfonate as the sole carbon source. The agar plates were incubated at $30^{\circ} \mathrm{C}$ for 10 days and the substrate range of each strain noted; the purity of cultures with a wide substrate range was confirmed by plating. Representative organisms were examined in liquid culture $(5 \mathrm{ml}$ in $30-\mathrm{ml}$ culture tubes) with either the appropriate mixture of substrates or with individual substrates.

\section{Results}

\section{Degradation of a mixture of sulfonated compounds by a defined co-culture}

The co-culture initially consisted of only two organisms, strains O-1 and PSB-4, with three carbon sources (2-aminobenzenesulfonate, 4-carboxybenzenesulfonate and 4-aminobenzenesulfonate), the latter of which could not be degraded by either strain. 50 Days after starting the continuous culture $\left(\mathrm{D}=0.004 \mathrm{~h}^{-1}\right)$ no organism had acquired the ability to degrade 4-aminobenzenesulfonate and no 4-aminobenzenesulfonate disappeared. In a similar experiment, Latorre et al. (1984) obtained a hybrid strain, which could degrade chloroaniline, through natural genetic exchange between an aniline degrader and a chlorobenzoate degrader.

Conditions in the chemostat were then altered by the addition of (a) benzenesulfonate and toluenesulfonate with $P$. testosteroni T-2 and strain S- 
1 (on day 58), and (b) 3-aminobenzenesulfonate and 4-hydroxybenzenesulfonate (on day 653) and strain M-1 (on day 685). After periods of adaption (21 days, $\mathrm{D}=0.010 \mathrm{~h}^{-1}$ ) to the new substrates the defined co-culture in the chemostat was able to mineralize all seven sulfonated compounds completely, as judged by HPLC (Fig. 1) and dissolved organic carbon analyses ( $>95 \%$ degradation).

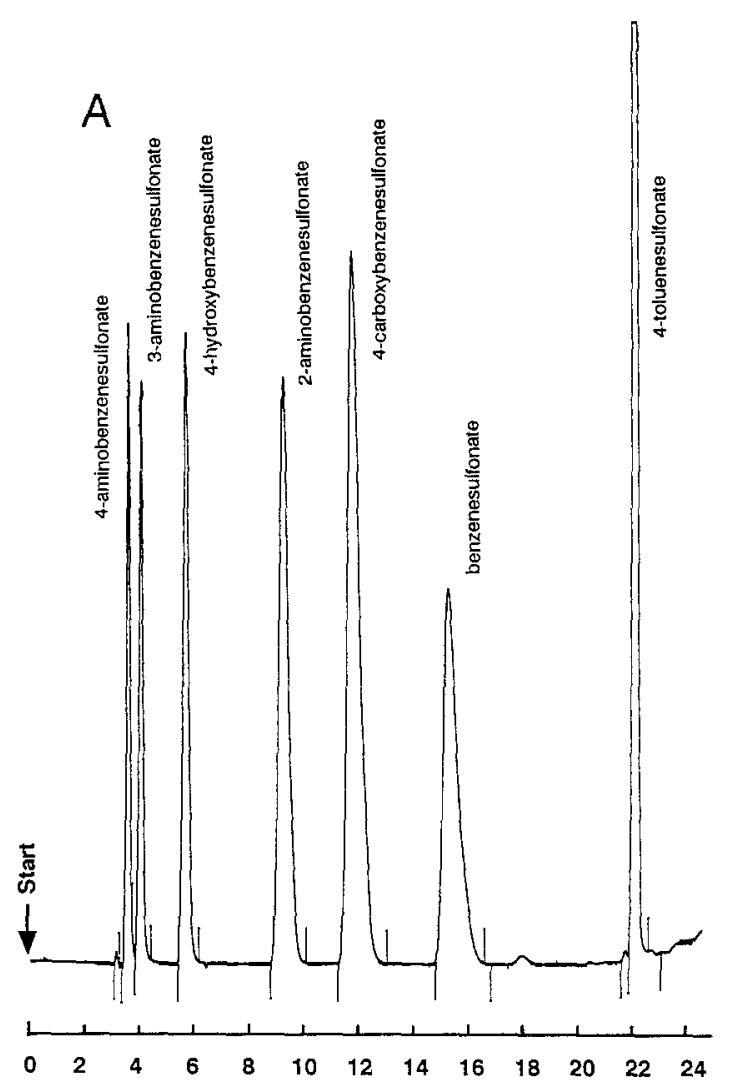

B

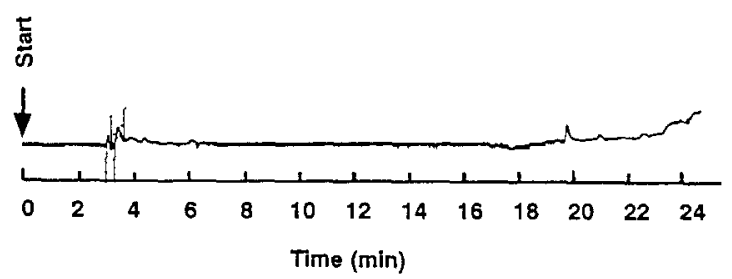

Fig. 1. HPLC-chromatograms of the co-culture in the chemostat. Tenfold diluted samples $(20 \mu \mathrm{l})$ were chromatographed with $100 \mathrm{mM}$ potassium phosphate buffer (pH 2.2). After 15 min a stepped gradient was applied to obtain $80 \%$ of the second solvent $(80 \%(\mathrm{v} / \mathrm{v})$ methanol in $10 \mathrm{mM}$ potassium phosphate buffer ( $\mathrm{pH} 2.2)$ ). $10 \mathrm{~min}$ later a second step to $100 \%$ of the second solvent was applied. The UV-detector was set at $220 \mathrm{~nm}$. The samples of feed (A) and effluent (B) were taken on day 730 (see Fig. 2)

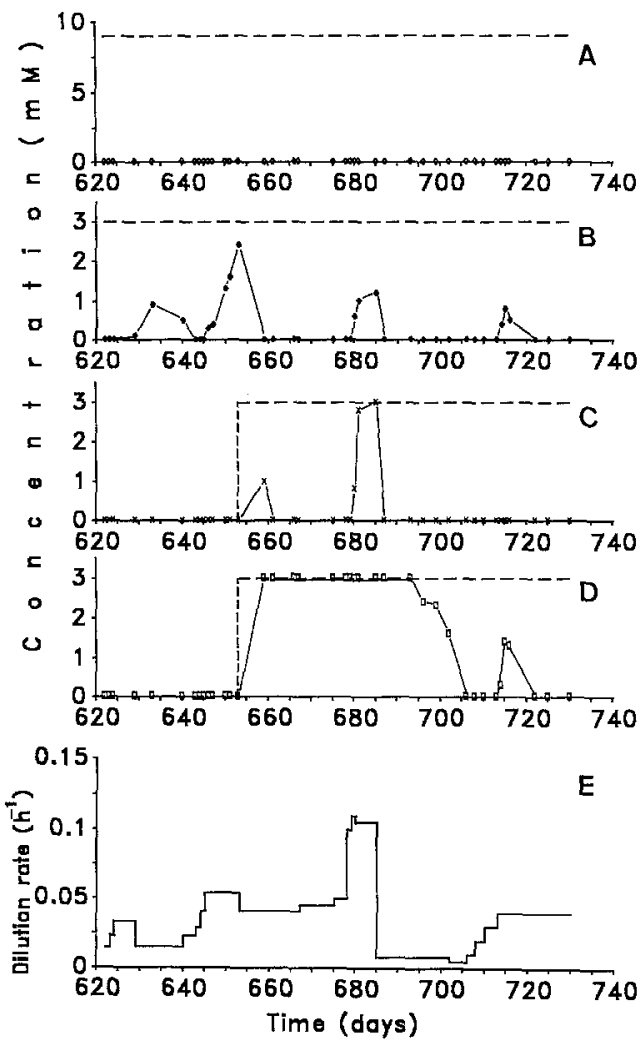

Fig. 2. Degradation of a mixture of 7 sulfonated compounds by the co-culture in a chemostat. (O) benzenesulfonate, 2-aminobenzenesulfonate, 4-sulfobenzoate and toluenesulfonate; (O) 4-aminobenzenesulfonate; $(x)$ 4-hydroxybenzenesulfonate; () 3-aminobenzenesulfonate; (---) concentration in feed $(\mathrm{mM}) ;(-)$ concentration in effluent $(\mathrm{mM})$

Typical responses of the co-culture to changes in the dilution rate and in the concentrations of substrates are shown in Fig. 2; for simplicity, four substrates namely benzenesulfonate, 2 -aminobenzenesulfonate, 4-carboxybenzenesulfonate and 4methylbenzenesulfonate, which all showed the same degradation pattern, are depicted in one diagram (Fig. 2A). The concentration of 4-aminobenzenesulfonate in the effluent increased with each increase in the dilution rate $(624,645,678$ and 714 days; Fig. 2B), but decreased again on further incubation. Utilization of 4-hydroxybenzenesulfonate needed only a short adaptation period ( 9 days from day 653; Fig. $2 \mathrm{C}$ ) and the compound was then totally degraded, presumably by strain S-1 (Table 1). None of the four organisms, which comprised the co-culture until day 685 , was able to degrade 3-aminobenzenesulfonate. Only after strain M-1 was added on day 685 could mineralization of this compound be observed (Fig. 2D). 
Table 2. Degradation rates observed in the chemostat

\begin{tabular}{llll}
\hline $\begin{array}{l}\text { Time } \\
\text { (days) }\end{array}$ & $\begin{array}{l}\text { Substrate concen- } \\
\text { tration in feed } \\
(\mathrm{g} \mathrm{C} / \mathrm{l})\end{array}$ & $\begin{array}{l}\text { Dilution } \\
\text { rate } \\
\left(\mathrm{h}^{-1}\right)\end{array}$ & $\begin{array}{l}\text { Degradation } \\
\text { rate } \\
(\mathrm{g} \mathrm{C} / \mathrm{h} \cdot \mathrm{l})\end{array}$ \\
\hline 96 & 2.09 & 0.006 & 0.012 \\
120 & 2.65 & 0.002 & 0.005 \\
175 & 2.50 & 0.003 & 0.008 \\
313 & 2.88 & 0.006 & 0.017 \\
330 & 2.95 & 0.015 & 0.044 \\
364 & 3.17 & 0.050 & 0.158 \\
415 & 3.39 & 0.014 & 0.047 \\
625 & 3.02 & 0.033 & 0.100 \\
645 & 3.02 & 0.041 & 0.124 \\
713 & 3.46 & 0.040 & $0.138^{\mathrm{b}}$ \\
730 & 3.46 & 0.040 & 0.138
\end{tabular}

a The substrates present from day 58 to day 653 were BS, $\mathrm{OS}, \mathrm{SB}$ and TS (up to $9 \mathrm{mM}$ each; see Table 1 for abbreviations) and $3 \mathrm{mM} \mathrm{SS}$. On day $653,3 \mathrm{mM}$ PS and $3 \mathrm{mM}$ MS were added. The concentration of sulfonated compounds in the corresponding effluent was below the detection limit of the HPLC $(10 \mu \mathrm{M})$

b The chemostat contained about $1.5 \mathrm{~g}$ of protein $/ 1$. The exact value could not be determined due to growth on the wall of the reaction vessel

The highest stable degradation rate, which we attained with the system, was $0.138 \mathrm{~g} \mathrm{C} / \mathrm{h} \cdot 1$ (Table 2). Incomplete degradation of benzenesulfonates due to apparatus malfunctions was observed e.g., growth of microorganisms on the $\mathrm{pH}$-electrode resulted in an incorrect $\mathrm{pH}$-value in the reaction vessel, and a punctured air line led to inadequate aeration. The system recovered quickly from such perturbations, and we noted that total biodegradation of the mixture at high degradation rates occurred only when the reaction vessel was well aerated, presumably because many of the enzymic reactions in the degradative pathways of substituted aromatics are oxygenases which require molecular oxygen.

At day 903, $3 \mathrm{mM}$ 4-chlorobenzenesulfonate was added to the co-culture. HPLC analysis 15 days later showed that this compound had also been degraded. Experiments are in progress to isolate the organism degrading 4-chlorobenzenesulfonate.

\section{Isolation from the co-culture of organisms with novel combinations of degradative abilities}

The organisms Alcaligenes denitrificans O-1, Pseudomonas testosteroni T-2, Pseudomonas testosteroni PSB-4 and strain S-1 were genetically stable for at least 220 days (Table 3 ). In the next 220 days, however, marked changes in the distribution
Table 3. Degradative abilities of organisms in the chemostat

\begin{tabular}{|c|c|c|c|c|}
\hline \multirow[t]{2}{*}{$\begin{array}{l}\text { Parent } \\
\text { organisms }\end{array}$} & \multirow[t]{2}{*}{ Degradative ability } & \multicolumn{3}{|c|}{$\begin{array}{l}\% \text { Distribution } \\
\text { after (days) }\end{array}$} \\
\hline & & 220 & 440 & 810 \\
\hline O-1 & $\mathrm{BS}+\mathrm{OS}+\mathrm{TS}$ & 19 & 22 & - \\
\hline PSB-4 & SB & 19 & 10 & 5 \\
\hline S-1 & SS + PS & 27 & 20 & - \\
\hline $\mathrm{T}-2$ & $\mathrm{SB}+\mathrm{TS}$ & 35 & 2 & 30.5 \\
\hline- & $\mathrm{OS}+\mathrm{SB}$ & - & 33 & 8.5 \\
\hline- & $\mathrm{BS}+\mathrm{OS}+\mathrm{TS}+\mathrm{SB}$ & - & 1 & 3 \\
\hline- & $\mathrm{MS}+\mathrm{OS}+\mathrm{PS}+\mathrm{SB}+\mathrm{TS}$ & - & - & $1^{\mathrm{a}}$ \\
\hline- & $\mathrm{BS}+\mathrm{MS}+\mathrm{OS}+\mathrm{SB}+\mathrm{TS}$ & - & - & $1.5^{\mathrm{a}}$ \\
\hline- & $\mathrm{BS}+\mathrm{OS}+\mathrm{PS}+\mathrm{SB}+\mathrm{SS}+\mathrm{TS}$ & - & - & $1^{\mathrm{a}}$ \\
\hline- & $\mathrm{BS}+\mathrm{MS}+\mathrm{OS}+\mathrm{PS}+\mathrm{SB}+\mathrm{TS}$ & - & - & $1^{\mathrm{a}}$ \\
\hline- & $\mathrm{BS}+\mathrm{MS}+\mathrm{OS}+\mathrm{PS}+\mathrm{SB}+\mathrm{SS}+\mathrm{TS}$ & - & - & $4^{\mathrm{a}}$ \\
\hline- & other combinations & - & 12 & 44 \\
\hline Total & & 100 & 100 & 99.5 \\
\hline
\end{tabular}

of the genes specifying sulfonate degradation were deduced from the new phenotypes observed; all four wild types were still present. After a further 370 days, during which 3-aminobenzenesulfonate and strain M-1 were added, major redistributions of genetic material occurred. All degradative capacities were still present, but three wild types were absent (M-1, O-1 and S-1) and organisms capable of degrading all seven substrates on plates had developed.

There was sometimes a discrepancy between the degradative capacity of an organism on plates and in liquid culture, whereby the substrate range on plates was always wider and the ability to utilize the sulfonate on plates was not lost on the passage through liquid culture. Growth and quantitative substrate utilization were scored in liquid culture, whereas colony formation was scored on plates, but we cannot explain the discrepancy. New strains tended to lose their degradative capacities on subculturing through non-selective media.

\section{Discussion}

Our mixed culture routinely metabolized all substrates completely, leaving negligible amounts of dissolved organic carbon in the medium (Fig. 1, Table 2). Incomplete metabolism was observed during transitions (Fig. 2), but the system recovered rapidly from changes in substrate concentrations and from e.g., changes in $\mathrm{pH}$ or $\mathrm{pO}_{2}$ caused by apparatus malfunctions. This incomplete meta- 
bolism seems to be at the level of the substrate, due to a temporary insufficiency of the degradative organisms, rather than being due to the excretion of toxic and dead-end products observed by e.g., Knackmuss (1983). Presumably desulfonation occurs early in the degradative pathways, leading to non-xenobiotic intermediates, in contrast to the excretion of toxic, chlorinated intermediates studied by Knackmuss (1983); a simple co-culture would probably fail to degrade mixtures of chloro- and methylaromatics.

The maximum consistent degradation rate, $138 \mathrm{mg} \mathrm{C} / \mathrm{h} \cdot 1$ (Table 2), compares favourably with other systems, though comparisons are usually only available for single substrates, e.g., dichloromethane $(184 \mathrm{mg} \mathrm{C} / \mathrm{h} \cdot 1$ in a fluidized bed reactor; Gälli 1987), pentachlorophenol (27 $\mathrm{mg} \mathrm{C} / \mathrm{h} \cdot \mathrm{l}$; Brown et al. 1986) and dichlorophenol (1.3 mg C/h.l; Beltrame et al. 1982); degradation of mixed substrates, $s$-triazine wastes, was less efficient $(80 \%)$ and slower $(26 \mathrm{mg} \mathrm{N} / \mathrm{h} \cdot 1$ in a fluidized bed reactor; Hogrefe et al. 1986).

Our co-culture (four organisms and six substrates) showed no visible interactions amongst the organisms up to day 220 (Table 3), each organism metabolizing its substrates unaffected by the presence of the other bacteria. At some point before day 440 , alterations in the phenotypes became evident, presumably representing horizontal transfer of genes amongst organisms. These transfers seem to have increased in magnitude with time, because, by day 810 , several parent strains were no longer detected (Table 3 ) though all degradative capacities were still present and organisms able to degrade the whole range of sulfonates had appeared.

Acknowledgements. We are grateful to Dr. M. Thüer, CibaGeigy AG. for the assays of dissolved organic carbon. We thank P. Arnold, A. Jenny, T. Lüthy and K. Wallimann for an analysis of organisms in the chemostat. This investigation was supported by a grant from the Swiss Federal Institute of Technology, Zürich.

\section{References}

Beltrame P, Beltrame PL, Carniti P, Pitea D (1982) Kinetics of biodegradation of mixtures containing 2,4-Dichlorophenol in a continuous stirred reactor. Water Res 16:429-433

Bretscher H (1981) Waste disposal in the chemical industry. In: Leisinger T, Cook AM, Hütter R, Nüesch J (eds) Mi- crobial degradation of xenobiotics and recalcitrant compounds. Academic Press, London, p 65

Brown EJ, Pignatello JJ, Martinson MM, Crawford RL (1986) Pentachlorophenol degradation: a pure bacterial culture and an epilithic microbial consortium. Appl Environ Microbiol 52:92-97

Bull AT, Slater HJ (eds) (1982) Microbial interactions and communities, vol. 1. Academic, London

Cook AM, Hütter R (1981) $s$-Triazines as nitrogen sources for bacteria. J Agric Food Chem 29:1135-1143

Czekalowski JW, Skarzynsky B (1948) The breakdown of phenols and related compounds by bacteria. J Gen Microbiol $2: 231-238$

Gälli R (1987) Biodegradation of dichloromethane in waste water using a fluidized bed bioreactor. Appl Microbiol Biotechnol 27:206-213

Ghisalba O (1983) Chemical wastes and their biodegradation - an overview. Experientia 39:1247-1257

Greenberg AE, Connors JJ, Jenkins D (eds) (1981) Standard methods for the examination of water and wastewater, 15th edn American Public Health Association, New York, p 383

Grossenbacher H, Thurnheer T, Zürrer D, Cook AM (1986) Determination of sulphonated azo dyestuffs and their bacterial metabolites by high-performance liquid chromatography. J Chromatogr 360:219-223

Herbert RB, Holliman FG (1964) Aeruginosin B - a naturally occurring phenazinesulphonic acid. Proc Chem Soc 1964: 19

Hogrefe W, Grossenbacher H, Cook AM, Leisinger T (1986) Biotreatment of $s$-triazine-containing wastewater in a fluidized bed reactor. Biotechnol Bioeng 28:1577-1581

Knackmuss H-J (1983) Xenobiotic degradation in industrial sewage: haloaromatics as target substrates. Biochem Soc Symp 48:173-190

Latorre J, Reineke W, Knackmuss H-J (1984) Microbial metabolism of chloroanilines: enhanced evolution by natural genetic exchange. Arch Microbiol 140:159-165

Leidner H, Gloor R, Wüest D, Wuhrmann K (1980) The influence of the sulphonic group on the biodegradability of n-alkanebenzenesulphonates. Xenobiotica $39: 47-56$

Liberman DF, Fink R, Schaefer F (1986) Biosafety and biotechnology. In: Demain AL, Solomon NA (eds) Manual of industrial microbiology and biotechnology, American Society for Microbiology, Washington, p 402

Malaney GW (1960) Oxidative abilities of aniline-acclimated activated sludge. J Water Pollut Control Fed 32:13001311

Ramos JL, Timmis K (1987) Experimental evolution of catabolic pathways of bacteria. Microbiol Sci 4:228-237

Swisher RD (1987) Surfactant biodegradation, 2nd edn, Marcel Dekker, New York

Symons JM, Del Valle-Rivera LA (1961) Metabolism of organic sulfonates by activated sludge. Eng Ext Ser Purdue Univ 109:555-571

Thurnheer T, Köhler T, Cook AM, Leisinger T (1986) Orthanilic acid and analogues as carbon sources for bacteria: growth physiology and enzymic desulphonation. J Gen Microbiol 132:1215-1220 